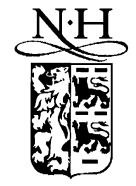

ELSEVIER

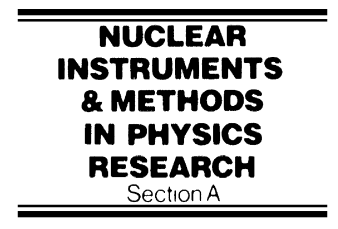

www.elsevier.nl/locate/nima

\title{
Uncertainty in radon measurements with CR39 detector due to unknown deposition of ${ }^{218} \mathrm{Po}$
}

\author{
D. Nikezić, K.N. Yu* \\ Department of Physics and Materials Science, City University of Hong Kong, Tat Chee Avenue, Kowloon Tong, Kowloon, Hong Kong
}

Received 23 August 1999; received in revised form 4 February 2000; accepted 9 February 2000

\begin{abstract}
Uncertainty in radon measurements using the CR39 solid-state nuclear track detector in chambers is introduced by the unknown fraction $f_{1}$ of ${ }^{218}$ Po that deposited onto the inner chamber walls. This uncertainty was estimated for cylindrical chambers with different size in the present study. It was found that the uncertainty increased with the chamber height and radius. For short chambers with a height $H=2 \mathrm{~cm}$ the uncertainty was less than $5 \%$, while in tall chambers with $H=7 \mathrm{~cm}$ it could be up to $17 \%$. The radial track density on the CR39 detector inside the diffusion chamber was also found to be dependent on the fraction $f_{1}$. In this work, some examples of this dependence are shown. (C) 2000 Elsevier Science B.V. All rights reserved.
\end{abstract}

PACS: $29.40 ; 23.60$

Keywords: Natural radioactivity; Radon; CR39 detector; Detector sensitivity; Monte Carlo methods

\section{Introduction}

For long-term passive measurements of indoor radon $\left({ }^{222} \mathrm{Rn}\right)$ concentrations solid-state nuclear track detectors (SSNTD) are frequently used. The most commonly used SSNTD is CR39, which is based on the polymer of alil diglycol carbonate. There are two different modes of its usage in radon measurements: (a) as open (bare) detector and (b) as detector in a diffusion chamber. There are different problems in both modes. In case of bare detector, humidity and dust affect the detection of alpha particles and the detector sensitivity is dependent

*Corresponding author. Tel.: + 852-2788-7812; fax: + 8522788-7830.

E-mail address: peter.yu@cityu.edu.hk (K.N. Yu). on the equilibrium factor between radon and its short-lived progeny. These were the reasons for the development and wide application of diffusion chambers for radon measurements.

The diffusion chamber is a cup covered with a filter paper on the top and is frequently equipped with a CR39 detector that is usually placed on the bottom of the cup. This technique is well described in the literature and used by many workers in the field. Radon gas diffuses through the filter paper into the chamber while its short-lived progeny are stopped by the filter paper. In this way, radon measurements are independent of the equilibrium factor between radon and its progeny. Radon gas atoms entering the chamber will decay in it and form progeny atoms. There is radioactive equilibrium between radon and its progeny inside the 
chamber. However, progeny atoms move randomly in the chamber volume and can deposit onto available inner surfaces before decay. The deposition changes the irradiation geometry of the detector, i.e., detector sensitivity is dependent on the fraction of radon progeny decaying in the air prior to deposition.

The behavior of radon progeny inside the chamber (as well as the aerosol properties and air velocity inside the chamber) is unknown and it is difficult to estimate the fraction of radon progeny decaying prior to deposition. The deposition fraction can depend on different environmental conditions (e.g., volume distribution of the electrical field inside the chamber), which influence the detector sensitivity and measurement accuracy.

The objective of the present work was to determine the uncertainty in radon measurements with the CR39 detector in diffusion chambers due to the unknown behavior of ${ }^{218} \mathrm{Po}$ inside the chambers. The dependence of the detector sensitivity on the distance from the center of the detector was also investigated.

The first radon progeny ${ }^{218} \mathrm{Po}$ has a relatively short half-life (3.05 $\mathrm{min})$ and it can partially decay before deposition. The second and third progeny in the radon decay chain, ${ }^{214} \mathrm{~Pb}$ and ${ }^{214} \mathrm{Bi}$, have much longer half-lives and we considered them as completely deposited. In this way, the second alphaemitting progeny in the radon chain, ${ }^{214} \mathrm{Po}$, was assumed as totally deposited. Therefore, the present work was limited to the study of the unknown behavior of ${ }^{218} \mathrm{Po}$ inside the chamber.

\section{Method and model}

The sensitivity $\varepsilon$ of the CR39 detector was defined as the number of tracks per unit surface area for an exposure to $1 \mathrm{~Bq} \mathrm{~m}^{-3}$ of some alpha emitter in air for $1 \mathrm{~s}$ (i.e. track density per unit exposure). The sensitivity $\varepsilon_{i}$ to the nuclide $i$ in the radon chain was called the partial sensitivity. The total sensitivity $\varepsilon_{\text {tot }}$ to the radon decay chain equals the sum of partial sensitivities to all alpha-emitting nuclides in the radon decay chain expressed as the ratio between the total number of tracks to the total surface of the detector:

$$
\begin{aligned}
\varepsilon_{\mathrm{tot}}= & \varepsilon_{\mathrm{Rn}-222}+f_{1} \varepsilon_{\mathrm{Po}-218}+\left(1-f_{1}\right) \varepsilon_{\mathrm{Po}-218}^{\prime}+\varepsilon_{\mathrm{Po}-214}^{\prime} \\
& +p_{1} N_{1}+p_{4} N_{4}
\end{aligned}
$$

where $\varepsilon_{\mathrm{Po}-218}$ is the sensitivity to ${ }^{218} \mathrm{Po}$ from air, $\varepsilon_{\text {Po-218 }}^{\prime}$ is the sensitivity to ${ }^{218}$ Po from the chamber wall and $\varepsilon_{\mathrm{Po}-214}^{\prime}$ is the sensitivity to ${ }^{214} \mathrm{Po}$ from the wall, $f_{1}$ is the part of ${ }^{218}$ Po that decayed in the chamber volume, $p_{i}$ the detection efficiency (in \%) for alpha-emitting progeny $\left(i=1\right.$ for ${ }^{218} \mathrm{Po}$, and $i=4$ for ${ }^{214} \mathrm{Po}$ ) deposited on the detector itself (or the so-called plate-out) and $N_{i}$ is the number of emitted alpha particles on the detector surface for these progeny for unit exposure.

\subsection{Geometrical model}

The geometry of the diffusion chamber considered in this work was cylindrical. A circular CR39 detector was situated at the bottom of the chamber so that the centers of the detector and the chamber base coincided with each other and the detector radius was equal to the radius of the chamber base. For this geometry, the detector covered the whole bottom of the chamber. Radon gas atoms were assumed to remain in air completely, i.e., no deposition. While ${ }^{218}$ Po was partially deposited, the other radon progeny decayed completely after deposition. A uniform deposition of radon progeny on all available surfaces of the chamber was also assumed.

\subsection{Calculation model}

Monte Carlo method was employed for the calculation of the partial sensitivities, and the total sensitivity was determined using Eq. (1). All calculations used $10^{4}$ histories. In addition, the radial sensitivity distribution on the detector was also determined. Calculations were made for the CR39 detector, with a thickness of $8 \mu \mathrm{m}$ for the removed layer.

The circular detector surface was divided into circular stripes with widths of $1 \mathrm{~mm}$ bounded by concentric circles. In the course of calculations, the number of alpha particles detected in different circular stripes was determined to deduce the radial sensitivity distribution. 
A previously developed program of Nikezic et al. [1] was used in these calculations. Details of the program were described earlier and will not be repeated here.

\section{Results}

Figs. 1a, b and c show the radial distribution of the total sensitivities for diffusion chambers with radius $r=2,3$ and 4 , respectively. Under these considerations the tracks from plateout are neglected. The chamber height was varied from 2 to $7 \mathrm{~cm}$, and the height is given as a parameter in Fig. 1. There are two groups of curves in the figures: the solid lines correspond to the assumption of $f_{1}=1$ (no deposition of ${ }^{218} \mathrm{Po}$ ) and the dashed lines to the assumption of $f_{1}=0$ (total deposition of ${ }^{218} \mathrm{Po}$ ). In all the cases shown in Fig. 1, the detector radius $r_{\text {det }}$ was the same as the chamber radius $r$, i.e., the detector covered the entire base of the chamber.

Fig. 1a gives the results for the chamber with a radius $r=2 \mathrm{~cm}$. The total sensitivities decrease from the center to the edge of the detector. This can be explained by the fact that the center of the detector was irradiated from all points above the detector while the edge was irradiated only from one side. This phenomenon is called the edge effect (or wall effect) in radon measurements with diffusion chambers. It can be observed that the ${ }^{218} \mathrm{Po}$ deposition decreased the sensitivity of CR39 detector to the radon chain by approximately $40 \%$.

Fig. $1 \mathrm{~b}$ gives the results for the $r=3 \mathrm{~cm}$ chamber. The total sensitivity obtained for $f_{1}=1$ decreased from the center towards the edge of the detector by a factor of slightly less than 2. However, the curves for $f_{1}=0$ were not monotonically decreasing (except the curve for a height $H=2 \mathrm{~cm}$ ). Instead, they showed weak and broad maximum values between 1 and $2 \mathrm{~cm}$ from the edge of the detector.

Fig. 1c gives the results for the $r=4$ chamber. The new feature in the curves of total sensitivities for $f_{1}=1$ was the relatively wide plateaus extending from the center up to $2 \mathrm{~cm}$ from the center, where the sensitivity is rather constant (with smaller variations). The curves for $f_{1}=0$ had peaks (except the curve for $H=2 \mathrm{~cm}$ ) close to the edge of the detector (about $1 \mathrm{~cm}$ from the end), which originated from the progeny deposited onto the wall. This peak is most expressed for tall chambers with $H=6$ and $7 \mathrm{~cm}$. Here, the possibility to study the ${ }^{218}$ Po deposition behavior inside diffusion chambers through the radial track density is demonstrated.

\subsection{Uncertainty}

Uncertainty $U$ is defined in the present work as

$U=\frac{\left|\bar{\varepsilon}-\varepsilon_{1}\right|}{\bar{\varepsilon}}$

where $\varepsilon_{1}$ and $\varepsilon_{2}$ are the total sensitivities calculated for $f_{1}=1$ and $f_{1}=0$, respectively, and $\bar{\varepsilon}=$ $\left(\varepsilon_{1}+\varepsilon_{2}\right) / 2$. The uncertainty in radon measurements introduced by an unknown $f_{1}$ has been calculated and shown in Fig. 2 as a function of the chamber height $H$, with the detector radius $R$ as a parameter (for $R=2,3$ and $4 \mathrm{~cm}$ ). The tracks originated by plateout are taken into account in the consideration of the uncertainties. It can be observed that the uncertainty increases with the chamber height. For the short chamber $(H=2 \mathrm{~cm})$ the uncertainty is rather small (below 5\%). For $H=3 \mathrm{~cm}$ the uncertainty is between $2 \%$ and $11 \%$. For tall chambers $(H=4$ to $7 \mathrm{~cm})$, the uncertainty ranges from $4 \%$ up to $16 \%$. The largest uncertainties were recorded in the chambers with $H=7 \mathrm{~cm}$, and range from $7 \%$ up to $16 \%$.

\section{Discussions}

${ }^{218} \mathrm{Po}$ atoms are formed in the unattached mode (or free mode) in diffusion chambers from the decay of ${ }^{222} \mathrm{Rn}$. The fate of these ${ }^{218} \mathrm{Po}$ atoms depends on the concentration and size distribution of aerosols inside the chamber. In general, the aerosol conditions inside a chamber are not known, and there are no data in the literature related to this question.

A method for determination of $f_{1}$ is proposed based on the results presented in this study. In large-volume chambers, e.g., $r=4, H=7$, the 

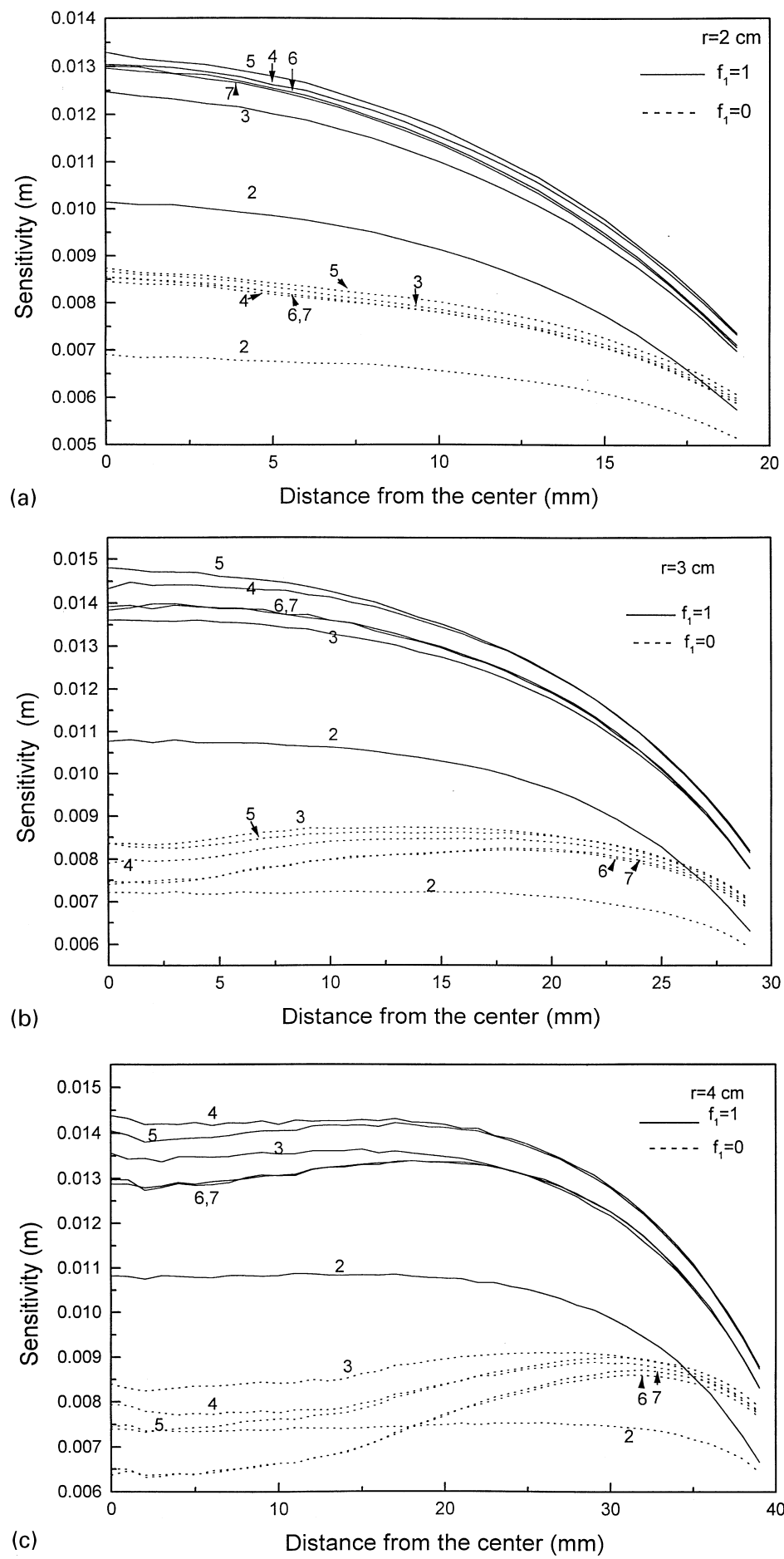

Fig. 1. Sensitivity distribution in diffusion chambers with different dimensions: (a) detector radius $r=2 \mathrm{~cm}$; (b) $r=3 \mathrm{~cm}$ and (c) $r=4 \mathrm{~cm}$. The numbers attached to the curves represent the height $(H)$ of the chambers. In all cases, the detector radius was the same as the chamber radius $R$, i.e., the detector covered the entire base of the chamber. The fraction of ${ }^{218}$ Po decayed before deposition is denoted as $f_{1}$. Plate-out tracks were neglected in these graphs. 


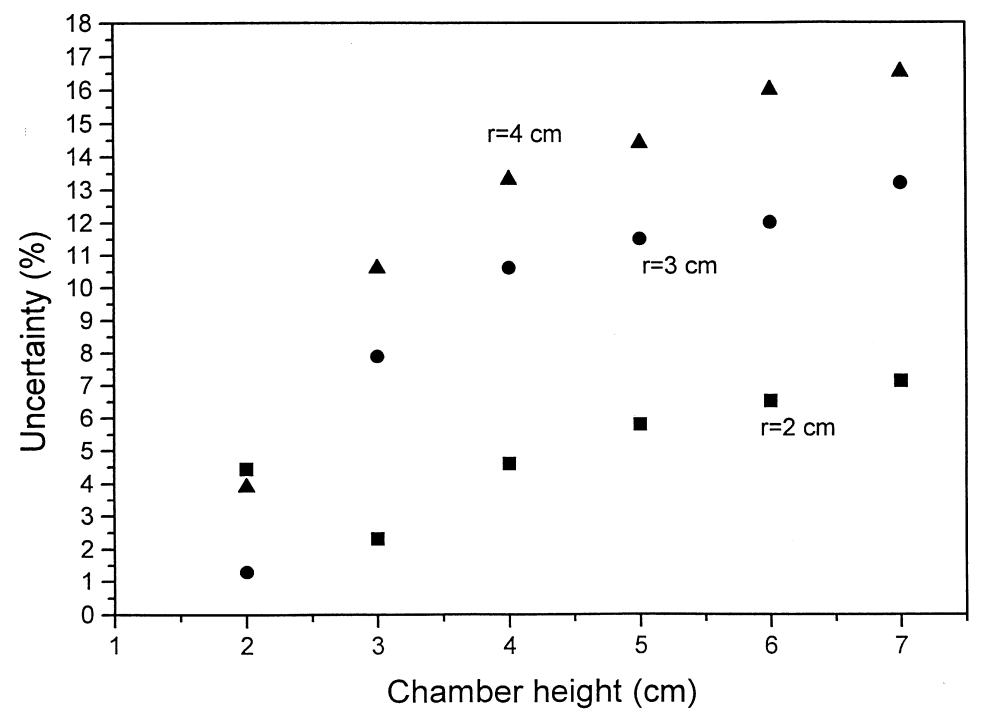

Fig. 2. Uncertainty in radon measurements with the CR39 detector (due to the unknown deposition behavior of ${ }^{218}$ Po inside the chambers) as a function of the chamber height. The results were given for chamber radii of 2,3 and $4 \mathrm{~cm}$.

deposited ${ }^{218}$ Po will lead to a peak in the radial track distribution close to the detector edge. The height of this peak is dependent on the deposition fraction of ${ }^{218} \mathrm{Po}$.

\section{Conclusions}

1. The radial track density distribution depends on the deposition fraction of ${ }^{218} \mathrm{Po}$. The dependence is more prominent in larger chambers, i.e., larger chambers are more suitable to be deployed to study the deposition of ${ }^{218} \mathrm{Po}$ inside diffusion chambers.

2. The size of the CR39 detector in the diffusion chamber is also important in radon measure- ments because the radial track density distribution and the detector sensitivity are also variable for different detector sizes.

3. The unknown deposition fraction of ${ }^{218} \mathrm{Po}$ introduces uncertainties in radon measurements using CR39 in diffusion chambers. The uncertainty depends on the dimensions of the chamber and detector, and the general trend is to increase with the chamber height. In large chambers, the uncertainty can be as high as $17 \%$.

\section{References}

[1] D. Nikezić, C. Baixeras, D. Kostić, Nucl. Instr. and Meth. A 373 (1996) 290. 